\title{
Concept of Gravito-Rotational Acceleration and its Consequences for Compact Stellar Objects
}

\author{
Mohamed E. Hassani ${ }^{1}$ \\ Institute for Fundamental Research \\ BP.197, CTR, Ghardaïa 47800, Algeria
}

\begin{abstract}
In a previous series of papers relating to the Combined Gravitational Action (CGA), we have exclusively studied orbital motion without spin. In the present paper, we apply CGA to any self-rotating material body, i.e., an axially spinning massive object, which itself may be locally seen as a gravito-rotational source because it is capable of generating the gravito-rotational acceleration, which seems to be unknown to previously existing theories of gravity. The consequences of such an acceleration are very interesting, particularly for Compact Stellar Objects. Independently of the equation of state, it is found that the critical and maximum internal magnetic field strength of a stable neutron star cannot exceed the value of $3 \times 10^{18} \mathrm{G}$.
\end{abstract}

Keywords: CGA; gravito-rotational acceleration; gravito-rotational energy; neutron stars; pulsars

PACS: $04.50 . \mathrm{Kd}, 45.20 . \mathrm{df}, 45.20 . \mathrm{dh}, 97.60 . J d, 97.60 . \mathrm{Gb}$

\section{Introduction}

\subsection{A brief summary of the CGA}

We feel that we are obliged to give a careful physical justification to the creation of the Combined Gravitational Action (CGA) as a refinement and a generalization of the Newton's gravity theory. The key idea in the CGA-formalism is the physical fact of taking into account the relative motion of the test(secondary)body which is under the gravitational influence of the primary one. Historically, the idea itself is not new since Laplace [1], Lorentz [2], Poincaré [3,4] and Oppenheim [5] have already thought of adjusting the Newton's law of gravitation by adding a certain velocity-dependent-term, but unfortunately their effort could not explain, e.g., the remaining secular perihelion advance rate of Mercury discovered by Le Verrier in 1859. We have previously shown in a series of articles [6-10] that the CGA as an alternative gravity theory is very capable of investigating, explaining and predicting, in its proper framework, some old and new gravitational phenomena. Conceptually, the CGA is basically founded on the concept of the combined gravitational potential energy (CGPE) which is actually a new form of velocity-dependent-GPE defined by the expression

$$
U \equiv U(r, v)=-\frac{k}{r}\left(1+\frac{v^{2}}{w^{2}}\right)
$$

where $k=G M m ; G$ being Newton's gravitational constant; $M$ and $m$ are the masses of the gravitational source $A$ and the moving test-body $B ; r=\sqrt{\left(x-x_{0}\right)^{2}+\left(y-y_{0}\right)^{2}+\left(z-z_{0}\right)^{2}}$ is the relative distance between $A$ and $B ; v=\sqrt{v_{x}^{2}+v_{y}^{2}+v_{z}^{2}}$ is the velocity of the test-body $B$ relative to the

\footnotetext{
1 E-mail: hassani641@gmail.com
} 
inertial reference frame of source $A$; and $w$ is a specific kinematical parameter having the physical dimensions of a constant velocity defined by

$$
w=\left\{\begin{array}{l}
c, \text { if } B \text { is in relative motion inside the vicinity of } A \\
v_{\text {esc }}=\sqrt{2 G M R^{-1}}, \text { if } B \text { is in relative motion outside the vicinity of } A
\end{array}\right.
$$

where $c$ is the light speed in local vacuum and $v_{\text {esc }}$ is the escape velocity at the surface of the gravitational source $A$.

It is worthwhile to note that the expression (1) constitutes a fundamental solution to a system of three second order PDEs, called 'potential equations' because $U \equiv U(r, v)$ is a common solution to these three equations. Indeed, it is easy to show that, under some appropriate boundary conditions, the combined potential field $U$ is a fundamental solution to the following equations:

$$
\left\{\begin{array}{l}
\frac{\partial^{2} U}{\partial r^{2}}+\frac{2}{r} \frac{\partial U}{\partial r}=0 \\
\frac{\partial^{2} U}{\partial v^{2}}-\frac{1}{v} \frac{\partial U}{\partial v}=0 \\
\frac{\partial^{2} U}{\partial r \partial v}+\frac{1}{r} \frac{\partial U}{\partial v}=0
\end{array}\right.
$$

In the CGA-context, the velocity-dependent-GPE (1) is simply called CGPE because it is, in fact, a combination of the static-GPE $V(r)=k r^{-1}$ and the dynamic-GPE $W(r, v)=k r^{-1}(v / w)^{2}$. The main difference between the CGPE (1) as a generalization of classical GPE and the previously well-known velocity-dependent-GPEs is clearly situated in the originality and simplicity of Eq.(1), which may be rewritten in the form $U \equiv U(r, \dot{r})=-k r^{-1}\left(1+(\dot{r} / w)^{2}\right)$, with $v \equiv \dot{r}=d r / d t$. The originality of CGPE is reflected by the fact that the CGPE is explicitly depending on $r$ and $v$ but also is implicitly depending on $w$ since the latter is, by definition, - a specific kinematical parameter having the physical dimensions of a constant velocity - . The implicit dependence of CGPE on $w$ is expressed in terms of 'inside the vicinity of $A$ ' and 'outside the vicinity of $A$ ' in (2). Furthermore, the CGPE may be reduced to the static-GPE when $v<<w$. Thus the main physical reason for the choice of the expression (1) for the CGPE lies in its consequence as a generalization of the static-GPE.

Now, let us show when and how we could apply the CGA. As we know, the Newton's gravitational theory is a very good depiction of gravity for many situations of practical, astronomical and cosmological interest. However, it is currently well established that the Newton's theory is only an approximate description of the law of gravity. As early as the middle of the nineteenth century, observations of the Mercury's orbit revealed a discrepancy with the prediction of Newton's gravity theory. In fact, this famous discrepancy was historically the first evidence of the limit of validity of Newtonian gravity theory. This disagreement between theory and observations was resolved by taking into account the CGA-effects inside and outside the solar system [6-9], which are known as the crucial tests support the general relativity theory (GRT). On the whole, the criterion that we should use to decide whether to employ Newton's gravity or CGA is the magnitude of a dimensionless physical quantity called the "CGA-correction factor" : 


$$
\zeta=G M / r c^{2} \cong v^{2} / c^{2}
$$

which is actually derived from (1) and (2) for the case when the test-body $B$ is evolving inside the vicinity of the gravitational source $A$. The same dimensionless physical quantity (4) exists in GRT and for this reason we have already shown in Ref.[10] the existence of an important similarity between the CGA-equations of motion and those of GRT. Moreover, it is worthwhile to note that the smaller this factor (4), the bitter is Newtonian gravity theory as an approximation. As an illustration, we have e.g., for the system $\{$ Earth, Moon $\}: \zeta \cong 10^{-11}$ and for the system $\{$ Sun, Earth $\}: \zeta \cong 10^{-8}$.

Hence, starting from the CGPE and using only the very familiar tools of classical gravitomechanics and the Euler-Lagrange equations, we have established the CGA-formalism [6-10] .The main consequence of CGA is the dynamic gravitational field (DGF), $\boldsymbol{\Lambda}$, which is phenomenologically an induced field that is more precisely a sort of gravitational induction due to the relative motion of material body inside the vicinity of the gravitational source [6-10]. In general, the magnitude of DGF is of the form

$$
\Lambda= \pm G M r^{-2}\left(\frac{v}{w}\right)^{2}
$$

Eq.(5) means that DGF may play a double role, that is to say, when perceived/interpreted as an extra-gravitational acceleration, $\Lambda>0$, or an extra-gravitational deceleration, $\Lambda<0$, (see Ref. [8] for a detailed discussion).

In the papers [6-10] we have exclusively focused our interest on the orbital motion and gravitational two-body problem. In the present paper, we shall apply CGA to any self-rotating (spinning) material body, i.e., axially rotating massive object that itself may be locally seen as a gravito-rotational source since it is capable of generating the gravito-rotational (field) acceleration $\lambda$, which seems to be unknown to previous theories of gravity.

\section{Concept of the Gravito-Rotational Acceleration}

Phenomenologically, the concept of the gravito-rotational acceleration (GRA), $\lambda$, is very similar to DGF, that is if $\boldsymbol{\Lambda}$ is mainly induced by the relative motion of the massive test body in the vicinity of the principal gravitational source, the GRA is intrinsically generated by any massive body in a state of rotational motion, independently of the principal gravitational source, which itself may be characterized by its proper GRA during its axial-rotation, and therefore the GRA is, in fact, a combination of gravity and rotation.

\section{Expression of GRA magnitude}

In order to derive an explicit expression for GRA magnitude, let us first rewrite Eq.(5) for the case when $\Lambda>0$, that is

$$
\Lambda=G M r^{-2}\left(\frac{v}{w}\right)^{2}
$$

and consider a massive body of mass $M$ and $\operatorname{radius} R$, which is intrinsically in a state of axial-rotation in its proper reference frame at rotational velocity of magnitude $v_{\text {rot }}=\Omega R$ independently of the 
presence of any other gravitational source. Therefore, according to the concept of GRA, in such a case, the rotating/spinning massive body should be locally seen as a gravito-rotational source when $\|\boldsymbol{\Lambda}\| \rightarrow\|\boldsymbol{\lambda}\| \equiv \boldsymbol{\lambda}$ as $r \rightarrow R, v \rightarrow v_{\text {rot }}$ and $w \rightarrow c$, thus (6) becomes after substitution

$$
\lambda=G M\left(\frac{\Omega}{c}\right)^{2} .
$$

Since $\Omega=2 \pi P^{-1}$, where $P$ is the rotational period, hence on insertion into (7), we get the expected expression

$$
\lambda=\kappa \frac{M}{P^{2}}, \quad \kappa=4 \pi^{2} G / c^{2}
$$

It is clear from Eq.(8), the GRA $\lambda$ depends exclusively on the mass and rotational period, therefore, mathematically may be treated as a function of the form $\lambda \equiv \lambda(M, P)$. Moreover, the structure of Eq.(8) allows us to affirm that for any astrophysical massive object, the magnitude of $\lambda$ should be infinitesimally small for slowly rotating massive stellar objects and enormous for rapidly rotating ones. To confirm our assertion numerically, we have selected seven well-known (binary) pulsars and calculated their GRA magnitudes, and compared them with the magnitude of the Sun's GRA. The values are listed in Table 1.

Table 1: The values of GRA magnitude for seven well-known (binary) pulsars compared with that of the Sun.

\begin{tabular}{lcccl}
\hline \hline $\begin{array}{c}\text { OвJест } \\
\text { Sun + PRS }\end{array}$ & $\begin{array}{c}P \\
(\mathrm{~s})\end{array}$ & $\begin{array}{c}M \\
\left(M_{\odot}\right)\end{array}$ & $\begin{array}{c}\lambda \\
\left(\mathrm{m} \mathrm{s}^{-2}\right)\end{array}$ & $\begin{array}{l}\text { REF. } \\
\text { Sun }\end{array}$ \\
\hline B 1913+16 & $2.164320 \times 10^{6}$ & 1 & $1.244823 \times 10^{-8}$ & \\
B 1534+12 & $5.903000 \times 10^{-2}$ & 1.4410 & $2.409380 \times 10^{7}$ & $\mathrm{a}$ \\
B 2127+11C & $3.790000 \times 10^{-2}$ & 1.3400 & $5.435171 \times 10^{7}$ & $\mathrm{~b}, \mathrm{c}$ \\
B 1257+12 & $6.200000 \times 10^{-3}$ & 1.4000 & $2.121932 \times 10^{9}$ & $\mathrm{~d}$ \\
J 0737-3039 & $2.280000 \times 10^{-2}$ & 1.3381 & $1.500000 \times 10^{8}$ & $\mathrm{f}$ \\
B 1937+21 & $1.557800 \times 10^{-3}$ & 1.4000 & $3.364000 \times 10^{10}$ & $\mathrm{~g}$ \\
J 1748-2446ad & $1.395000 \times 10^{-3}$ & 1.4000 & $4.194982 \times 10^{10}$ & $\mathrm{~h}$ \\
\hline
\end{tabular}

Ref.: a) Taylor and Weisberg [11]; b) Arzoumanian [12]; c) Wolszcan [13]; d) Deich and Kulkarni [14];

e) Konacki and Wolszcan [15]; f) Kramer and Wex [16]; g) Takahashi et al. [17];

h) Hessels et al. [18].

Note: To calculate these values, we have used $G=6.67384 \times 10^{-11} \mathrm{~m}^{3} \mathrm{~kg}^{-1} \mathrm{~s}^{-2}, c=299792458 \mathrm{~m} \mathrm{~s}^{-1}$,

$M_{\odot}=1.9885 \times 10^{30} \mathrm{~kg}$ and sidereal rotation period at equator $P_{\odot}=25.05 \mathrm{~d}$. 
Analysis of Table1 gives us the following results: 1) The magnitude of the Sun's GRA, $\lambda_{\odot}=1.244823 \times 10^{-8} \mathrm{~ms}^{-2}$, is extremely weak that's why its effect on the solar system is unobservable, but perhaps it is only the Sun's immediate vicinity that should be affected by it. Since GRA is explicitly independent of the radius of the rotating massive object thus the extreme weakness of the magnitude of the Sun's GRA is mainly due to the huge value of the rotational period, $P_{\odot}$ $=2.164320 \times 10^{6} \mathrm{~s}$, compared with those of the pulsars. 2) In spite of the fact that the pulsars' masses are nearly equal, the pulsars' rotational periods show a neat inequality between them. Also, the different values of GRA magnitude for each celestial object show us how sensitive GRA is to variation in rotational period.

\section{Mutual dependence between the Mass and the Rotational Period}

Since GRA may be treated as a function of the form $\lambda \equiv \lambda(M, P)$ thus we can show more clearly the existence of the mutual dependence between the mass and rotational period of the same rotating body via GRA. For this purpose, we deduce from Eq.(8) the following expression

$$
\frac{M}{P^{2}}=\kappa^{-1} \lambda
$$

Obviously, Eq.(9) shows us the expected mutual dependence between the mass and rotational period via GRA. Furthermore, as the rotational period is an intrinsic physical quantity, here, according to Eq.(9), the spin of any massive celestial body should vary with mass independently of cosmic time.

\section{Link between GRA and Rotational Acceleration}

Now, returning to Eq.(8) and showing that GRA and the rotational acceleration

$$
a_{\mathrm{rot}}=\Omega^{2} R,
$$

are in fact proportional, $\lambda \propto a_{\mathrm{rot}}$, and the constant of proportionality is precisely the compactness factor $\varepsilon=G M / c^{2} R$ that characterizes any massive celestial body. To this end, it suffices to multiply and divide by the radius $R$ the right-hand side of Eq.(8) to get the expected expression

$$
\lambda=\varepsilon a_{\mathrm{rot}} .
$$

According to the expression (11), GRA is at the same time an old and a new natural physical quantity that should play a crucial role, especially for compact stellar objects, e.g., the rotating neutron stars and pulsars for which the compactness $\varepsilon$ has a large value compared to that of normal stellar objects. By way of illustration, the Sun's compactness $\varepsilon_{\odot}$ has the value of $2.123679 \times 10^{-6}$. 


\section{Consequences of GRA}

In what follows we will show that, in the CGA-context, the gravitodynamic equilibrium, dynamical instability and gravitational instability of a uniformly rotating neutron star (NS) depend on the 'antagonism' between centrifugal force and gravitational force, or in energetic terms, between rotational kinetic energy (RKE) and gravitational binding energy (GBE).

Usually the physics of NS/pulsar considers that the source of the emitted energy is essentially the RKE, however, such a consideration should immediately imply that, at least in the medium term, the GBE should absolutely dominate the RKE and as a result the NS should be prematurely in a state of gravitational collapse. Hence, as we will see, the main source of the emitted energy is not the RKE but the gravito-rotational energy (GRE), a sort of new physical quantity which is a direct consequence of GRA.

Let us now determine the states of gravitodynamic equilibrium, dynamical instability and gravitational instability that may be characterized any NS at least in the medium term. With this aim, we assume a uniformly rotating NS as a homogeneous rigid spherical body of mass $M$, radius $R$ and angular velocity $\Omega=2 \pi P^{-1}$, where $P$ is the rotational period. The NS's RKE and GBE are, respectively, defined by the well-known formulae:

$$
\begin{aligned}
E_{\mathrm{rot}} & =\frac{1}{2} I \Omega^{2}, \\
E_{\mathrm{g}} & =-\frac{3}{5} G M^{2} R^{-1},
\end{aligned}
$$

where $I=\frac{2}{5} M R^{2}$ is the moment of inertia of NS under consideration. Hence, the total energy is

$$
E_{\mathrm{tot}}=E_{\mathrm{rot}}+E_{\mathrm{g}}
$$

which presents the following three states according to the sign of total energy:

a) $E_{\text {tot }}<0, \mathrm{NS}$ is in a state of gravitational instability,

b) $E_{\text {tot }}=0, \mathrm{NS}$ is in a state of gravitodynamic equilibrium,

c) $E_{\text {tot }}>0, \mathrm{NS}$ is in a state of dynamical instability.

It is worth noting that the three suggested states a), b) and c) are taken in the medium term because NS may be suddenly in a state of gravitational or dynamical perturbations or in a state of transition from temporary stability to temporary instability and vice versa.

\section{Critical Rotational Period}

Knowing the critical rotational period (CRP) of NS is very important because CRP should be treated as a parameter of reference on which the temporal evolution of NS depends. Furthermore, since the change from temporary stability to temporary instability and vice versa should pass obligatorily via the state of gravitodynamic equilibrium, therefore, an expression for the CRP may be deduced from 
the state (b), so after performing a simple algebraic calculation, we get the following expected expression

$$
P_{\mathrm{c}}=2 \pi R \sqrt{\frac{R}{3 G M}}
$$

If we take into account the relativistic considerations, the realistic critical rotational period should be

$$
P_{\mathrm{c}} \geq \frac{2 \pi R}{c}
$$

From (15) and (16) we obtain an expression for minimum radius of any stable NS

$$
R_{\min }=\frac{3 G M}{c^{2}} .
$$

Eq.(17) asserts that the minimum radius $R_{\min }$ of any stable NS of mass $M$ is three times the gravitational radius $r_{\mathrm{g}}=G M / c^{2}$ of the NS under consideration. Now, let us calculate the $P_{\mathrm{c}}, \lambda_{\mathrm{c}}$ and $R_{\text {min }}$, respectively, of standard NS $\left(M=1.4 M_{\odot}, R=10 \mathrm{~km}\right)$. On substitution, we have

$$
\begin{aligned}
P_{\mathrm{c}} & =2.661365 \times 10^{-4} \mathrm{~s}=266.1365 \mu \mathrm{s}, \\
\lambda_{\mathrm{c}} & =1.152 \times 10^{12} \mathrm{~ms}^{-2}, \\
R_{\text {min }} & =6.201678 \mathrm{~km} .
\end{aligned}
$$

It is worthwhile to note that the calculated critical rotational period (18) for the standard NS is a tiny fraction of the smallest yet observed rotational period, $P=1.3950 \mathrm{~ms}$, of PRS J1748-2446ab [13].

\section{Gravito-Rotational Energy}

Now we approach the most important consequence of GRA, that is, the gravito-rotational energy (GRE), which should qualitatively and quantitatively characterize any massive rotating body. As we will see, GRE is quantitatively very comparable to the amount of RKE, particularly for neutron stars and pulsars. Since GRE is a direct consequence of GRA, hence GRE should be proportional to GRA magnitude, i.e., $E \propto \lambda$ or equivalently

$$
\varepsilon=\eta \lambda
$$

Let us determine the constant of the proportionality $\eta$ by using dimensional analysis as follows:

$$
[\eta]=\frac{[\mathscr{E}]}{[\lambda]}=\frac{\mathrm{ML}^{2} \mathrm{~T}^{-2}}{\mathrm{LT}^{-2}}=\mathrm{ML} \text {. }
$$

we can remark that the dimensional quantity $\mathrm{ML}$ has the physical dimensions of the product of mass and length, therefore, for our case $\eta$ should take the form $\eta=M R=5 I / 2 R$ and by substituting into (21), we find the required expression for GRE 


$$
\varepsilon=\frac{5}{2} I \lambda R^{-1}
$$

In order to show that the amount of GRE $\varepsilon$ is quantitatively very comparable to that of RKE, particularly for the compact stellar objects, we can use Table 1 . The numerical values of $E_{\text {rot }}$ and $\varepsilon$ are listed in Table 2.

Table 2: Comparison of the numerical values of $E_{\text {rot }}$ and $\varepsilon$ for the Sun and seven well known (binary) pulsar

\begin{tabular}{lcc}
\hline \hline $\begin{array}{c}\text { Ов JECт } \\
\text { Sun + PRS }\end{array}$ & $\begin{array}{c}E_{\text {rot }} \\
(\mathrm{J})\end{array}$ & $\begin{array}{c}\mathcal{E} \\
(\mathrm{J})\end{array}$ \\
\hline Sun & $2.3900 \times 10^{35}$ & $2.5401 \times 10^{30}$ \\
B 1913+16 & $6.4948 \times 10^{41}$ & $6.9180 \times 10^{41}$ \\
B 1534+12 & $1.4651 \times 10^{42}$ & $1.4500 \times 10^{42}$ \\
B 2127+11C & $2.2915 \times 10^{42}$ & $2.3010 \times 10^{42}$ \\
B 1257+12 & $5.7200 \times 10^{43}$ & $5.9140 \times 10^{43}$ \\
J 0737-3039 & $4.0426 \times 10^{42}$ & $4.0000 \times 10^{42}$ \\
B 1937+21 & $9.0604 \times 10^{44}$ & $9.3680 \times 10^{44}$ \\
J 1748-2446ad & $1.1300 \times 10^{45}$ & $1.1680 \times 10^{45}$ \\
\hline \hline
\end{tabular}

Note: To calculate the Sun's $E_{\text {rot }}$ and $\mathcal{E}$, we have used the relation $I / M R^{2}=0.059$.

Analysis of Table 2: The numerical values listed in Table 2 show us, excepting the Sun's values, that all the values of $E_{\text {rot }}$ and $\&$ are very comparable for the seven (binary) pulsars. This fact is mainly due, at the same time, to the rotational period and the compactness $\varepsilon$. To illustrate this fact, let us return to the expression (22) which may be written as follows:

$$
\varepsilon=5 \varepsilon E_{\text {rot }} \text {. }
$$

And as $5 \varepsilon \cong 1$ for the NS hence that's why $\mathscr{E} \cong E_{\text {rot }}$ as it is well illustrated in Table 2. From all that we arrive at the following result: In the CGA-context, the RKE cannot be considered as the main source of the emitted energy for rotating neutron stars and pulsars because -in energetic terms - its own role is to balance, approximately, the GBE, at least in the medium term. Therefore, the veritable principal source of the emitted energy should undoubtedly be GRE, as illustrated by the GRE numerical values listed in Table 2, which are quantitatively very comparable to those of RKE for pulsars. Moreover, if we take into consideration the critical value of GRA magnitude (19) for standard NS $\left(M=1.4 M_{\odot}\right.$, $R=10 \mathrm{~km}$ ), we get the following critical value for GRE

$$
\varepsilon_{\mathrm{c}}=3.210 \times 10^{46} \mathrm{~J}=3.210 \times 10^{53} \mathrm{erg} .
$$




\section{Rotating Magnetars and Neutron stars}

Rotating magnetized neutron stars (magnetars) are also important compact stellar objects. That's why it is possible, in the CGA-context, to exploit GRE as an energetic reservoir for rotating magnetars by assuming that there is a certain physical mechanism that can convert all or at least a significant part of GRE into an extreme internal magnetic energy:

$$
E_{B}=\left(\frac{1}{8 \pi} B^{2}\right)\left(\frac{4}{3} \pi R^{3}\right)=\mathcal{E}(\mathrm{erg})
$$

By taking into account the expressions (7), (22) and after some algebraic manipulation, we get from (25) the expected equations for the internal magnetic field (IMF) strength

$$
B=k \frac{M}{R} \frac{\Omega}{c},
$$

or equivalently

$$
B=k \frac{M}{R^{2}} \frac{v_{\text {rot }}}{c}
$$

where

$$
k=6.328 \times 10^{-4} \mathrm{~cm}^{3 / 2} \mathrm{~g}^{-1 / 2} \mathrm{~s}^{-1}, \Omega=2 \pi P^{-1}, v_{\text {rot }}=\Omega R
$$

As we can see from Eq.(26), the IMF strength is also depending on the NS' angular velocity $\Omega$. This dependency has not yet been mentioned in the literature. This dependency will offer profound insight on the physics of neutron stars. Moreover, Eq.(27) permits us to calculate the theoretical maximum IMF strength of any NS independently of the equation of state (EOS), i.e., $B \rightarrow B_{\max }$ when $v_{\text {rot }} \rightarrow c$, or more explicitly:

$$
B_{\mathrm{max}}=k\left(\frac{M}{R^{2}} \frac{v_{\mathrm{rot}}}{c}\right)_{v_{\mathrm{rot}}=c} .
$$

Now, let us demonstrate that the same IMF strength defined by (27) may be treated as a function of surface gravity $g_{0}$ and rotational velocity of NS. To this end, multiplying and dividing the right-hand side of (27) by the same quantity $G$, Newton's gravitational constant, to obtain $B=K g_{0} v_{\text {rot }} c^{-1}$ where $K=9.482 \times 10^{3} \mathrm{~cm}^{-3 / 2} \mathrm{~g}^{1 / 2} \mathrm{~s}^{1}$ and $g_{0}=G M R^{-2}$.

Obviously, the surface magnetic field strength $B_{0}$ and average IMF strength $B_{\text {av }}$ should be, respectively, less than critical IMF strength $B_{\mathrm{c}}$ and maximum IMF strength $B_{\max }$. As an illustration, let us apply our formalism to a massive NS. To be realistic, we have selected the well-known pulsar PSR J1614-2230. So, we have according to [19] the following parameters: $P=3.15 \mathrm{~ms}, \quad M=1.97 M_{\odot}$, $R=13 \mathrm{~km}$ and $B_{0} \cong 1.8 \times 10^{8} \mathrm{G}$. Thus, by inserting these physical parameters into Eqs.(15), (26) and (28), we obtain the average, critical and maximum IMF strength, respectively: 


$$
\begin{aligned}
B_{\mathrm{av}} & =1.268713 \times 10^{17} \mathrm{G}, \\
B_{\mathrm{c}} & =1.202 \times 10^{18} \mathrm{G}, \\
B_{\max } & =1.466802 \times 10^{18} \mathrm{G} .
\end{aligned}
$$

Since, according to the present formalism which is established independently of EOS, the critical IMF strength (30) is calculated when the NS (PSR J1614-2230) is supposed to be in a state of gravitodynamic equilibrium $E_{\mathrm{tot}}=0$, i.e., state (b) by using Eqs.(15) and (26), therefore, it follows that any NS of mass $2 M_{\odot} \lesssim M_{\mathrm{NS}} \lesssim 3 M_{\odot}$ and radius $10 \mathrm{~km} \leq R_{\mathrm{NS}} \leq 15 \mathrm{~km}$ should be in a state of dynamical instability if its IMF strength is greater than the critical field $\left(B>B_{\mathrm{C}}\right)$ because the numerical value of maximum IMF strength (31) for PSR J1614-2230 is calculated for the case $v_{\text {rot }}=c$. On the whole, we can affirm, in the context of the present work, that any stable NS should be characterized by a critical $B_{\mathrm{c}}$ and a maximum IMF strength $B_{\max }$ of the order of $10^{18} \mathrm{G}$. To confirm our claims about this value, let us evaluate the critical and maximum IMF strength of three idealized stable neutron stars of masses: $2 M_{\odot} ; 2.5 M_{\odot} ; 3 M_{\odot}$ and radii: $10 \mathrm{~km} \leq R_{\mathrm{NS}} \leq 15 \mathrm{~km} ; 11.50 \mathrm{~km} \leq R_{\mathrm{NS}} \leq 15 \mathrm{~km}$; $13.50 \mathrm{~km} \leq R_{\mathrm{NS}} \leq 15 \mathrm{~km}$, respectively. Note that the idealized minimum radii $10 \mathrm{~km} ; 11.50 \mathrm{~km}$; $13.50 \mathrm{~km}$ are relatively comparable to the theoretical minimum radii $8.86 \mathrm{~km} ; 11.075 \mathrm{~km} ; 13.30 \mathrm{~km}$ computed with the help of Eq.(17). Like before, with the help of Eqs.(15), (26) and (28), the predicted values for each NS are computed and listed in Tables 3, 4 and 5.

Table 3: The values of critical and maximum IMF strength for an idealized stable NS of mass $2 M_{\odot}$

\begin{tabular}{cccc}
\hline \hline $\mathbf{N S}$ & $\begin{array}{c}R_{\mathrm{NS}} \\
(\mathrm{km})\end{array}$ & $\begin{array}{c}B_{\mathrm{c}} \\
(\mathrm{G})\end{array}$ & $\begin{array}{c}B_{\max } \\
(\mathrm{G})\end{array}$ \\
\hline$\left.M_{\odot}\right)$ & 10 & $2.368795 \times 10^{18}$ & $2.516645 \times 10^{18}$ \\
2 & 10.50 & $2.096787 \times 10^{18}$ & $2.282671 \times 10^{18}$ \\
2 & 11 & $1.866577 \times 10^{18}$ & $2.079872 \times 10^{18}$ \\
2 & 11.50 & $1.670256 \times 10^{18}$ & $1.902945 \times 10^{18}$ \\
2 & 12 & $1.501670 \times 10^{18}$ & $1.747670 \times 10^{18}$ \\
2 & 12.50 & $1.355977 \times 10^{18}$ & $1.610653 \times 10^{18}$ \\
2 & 13 & $1.229332 \times 10^{18}$ & $1.489139 \times 10^{18}$ \\
2 & 13.50 & $1.118647 \times 10^{18}$ & $1.380875 \times 10^{18}$ \\
2 & 14 & $1.021427 \times 10^{18}$ & $1.284002 \times 10^{18}$ \\
2 & 14.50 & $9.356375 \times 10^{17}$ & $1.196977 \times 10^{18}$ \\
2 & 15 & $8.596061 \times 10^{17}$ & $1.118509 \times 10^{18}$ \\
2 & & & \\
\hline
\end{tabular}


Table 4: The values of critical and maximum IMF strength for an idealized stable NS of mass $2.5 M_{\odot}$

\begin{tabular}{cccc}
\hline \hline $\mathbf{N S}$ & $R_{\mathrm{NS}}$ & $B_{\mathrm{c}}$ & $B_{\max }$ \\
$\left(M_{\odot}\right)$ & $(\mathrm{Gm})$ & $(\mathrm{G})$ \\
\hline 2.5 & 11.50 & $2.334253 \times 10^{18}$ & $2.378682 \times 10^{18}$ \\
2.5 & 12 & $2.098647 \times 10^{18}$ & $2.184588 \times 10^{18}$ \\
2.5 & 12.50 & $1.895036 \times 10^{18}$ & $2.013316 \times 10^{18}$ \\
2.5 & 13 & $1.718044 \times 10^{18}$ & $1.861424 \times 10^{18}$ \\
2.5 & 13.50 & $1.563357 \times 10^{18}$ & $1.720694 \times 10^{18}$ \\
2.5 & 14 & $1.427488 \times 10^{18}$ & $1.605003 \times 10^{18}$ \\
2.5 & 14.50 & $1.307593 \times 10^{18}$ & $1.496222 \times 10^{18}$ \\
2.5 & 15 & $1.201336 \times 10^{18}$ & $1.398136 \times 10^{18}$ \\
\hline \hline
\end{tabular}

Table 5: The values of critical and maximum IMF strength for an idealized stable NS of mass $3 M_{\odot}$

\begin{tabular}{clcc}
\hline \hline $\mathbf{N S}$ & $R_{\mathrm{NS}}$ & $B_{\mathrm{c}}$ & $B_{\max }$ \\
$\left(M_{\odot}\right)$ & $(\mathrm{Gm})$ & $2.055086 \times 10^{18}$ & $2.071313 \times 10^{18}$ \\
\hline 3 & 13.50 & $1.876481 \times 10^{18}$ & $1.926002 \times 10^{18}$ \\
3 & 14 & $1.718815 \times 10^{18}$ & $1.795466 \times 10^{18}$ \\
3 & 14.50 & $1.579196 \times 10^{18}$ & $1.677763 \times 10^{18}$ \\
3 & 15 & & \\
\hline
\end{tabular}

Close examination of Tables 3, 4 and 5 indicates that the critical and maximum IMF strength of an idealized stable $\mathrm{NS}$ of mass $2 M_{\odot} \leq M_{\mathrm{NS}} \leq 3 M_{\odot}$ and radius $10 \mathrm{~km} \leq R_{\mathrm{NS}} \leq 15 \mathrm{~km}$ is of the order of $10^{18} \mathrm{G}$ and, as a result, the critical and maximum IMF strength cannot exceed the value of $3 \times 10^{18} \mathrm{G}$. Very similar result has already been reported in Refs.[20, 21]. Accordingly, by taking into account of Eq.(26), the NS' mass density should be redefined, i.e., it becomes a function of the form $\rho \equiv \rho(B, \Omega, R)$ or more explicitly

$$
\rho=\frac{3}{4 \pi k} \frac{B c}{\Omega R^{2}} .
$$

Furthermore, according to (32), the EOS and the sound velocity should be redefined because both may be significantly affected by the IMF particularly when $B \geq B_{\mathrm{c}}$. 


\section{Conclusion}

Basing on our gravity model, Combined Gravitational Action, we have derived an explicit expression for the concept of gravito-rotational acceleration (GRA), which is unknown to previously established gravity theories. The most significant result of GRA is the gravito-rotational energy (GRE), which should qualitatively and quantitatively characterize any massive rotating body. Furthermore, GRE is exploited as an energetic reservoir, particularly for neutron stars and pulsars. Independently of the equation of state, it is found that the internal magnetic field of a stable neutron star cannot exceed the value of $3 \times 10^{18} \mathrm{G}$.

\section{References}

[1] P. S. Laplace, Traité de Mécanique Céleste, Tome IV, Livre X, Chap.7. Courcier, Paris (1805)

[2] H. A. Lorentz, Proceedings of the Royal Netherlands Academy of Arts and Sciences 2,559 (1900)

[3] H. Poincaré, C.-R. Acad. Sci. Paris 140,1504 (1905)

[4] H. Poincaré, Rendiconti Circ. Matemat. Palermo 21,129 (1906)

[5] S. Oppenheim, Ann. d. Phys. 52, 415 (1917)

[6] M. E. Hassani, Galilean Electrodynamics 20, Special Issues 3, 54 (2009)

[7] M. E. Hassani, Galilean Electrodynamics 22, Special Issues 3, 43 (2011)

[8] M. E. Hassani, Galilean Electrodynamics 26 (6), 103 (2015)

[9] M.E. Hassani, Galilean Electrodynamics 27 (1), 3 (2016)

[10] M. E. Hassani, vixra:1503.0143 (21 Sept. 2015)

[11] J. H. Taylor and J.M. Weisberg, ApJ, 345, 434 (1989)

[12] Z. Arzoumanian, PhD. Thesis, Princeton University (1995)

[13] A. Wolszczan, Nature, 350, 688 (1991)

[14] W. T.S.Deich and S.R.Kulkarni, in Compact Stars in Binaries: IAU. Symp.165, J.van Paradijs, E.P.J .van den Heuvel and E.Kuulkers, eds. (Dordrecht: Kluwer, 1996)

[15] M. Konacki and A. Wolszczan, ApJ, 591, L147 (2003)

[16] M. Kramer and N. Wex, Class. Quantum Grav. 26, 073001 (2009)

[17] M. Takahashi et al., ApJ, 554, 316 (2001)

[18] J. W.T. Hes sels et al., arXiv: as tro-ph/0601337 (16 January 2006)

[19] P. Demorest et al., Nature, 467, 1081 (2010)

[20] D. Lai and S. Shapiro ApJ, 383, 745 (1991)

[21] A. E. Broderick, et al., Phys. Lett. B 531, 167 (2002) 